\title{
On a q-analogue degenerate Carlitz's type Daehee polyno- mials and numbers
}

\author{
Jongkyum Kwon ${ }^{\mathrm{a}}$, Yunjae Kim ${ }^{\mathrm{b}}$, Gyoyong Sohn ${ }^{\mathrm{c}}$, Jin-Woo Park ${ }^{\mathrm{d}, *}$ \\ ${ }^{a}$ Department of Mathematics Education and ERI, Gyeongsang National University, Jinju, 52828, Republic of Korea. \\ ${ }^{b}$ Department of Mathematics, Dong-A University, Busan, 49315, Republic of Korea. \\ ${ }^{c}$ Department of Mathematics Education, Daegu National University of Education, Daegu, 705-715, Republic of Korea. \\ ${ }^{d}$ Department of Mathematics Education, Daegu University, Gyeongsangbuk-do, 38453, Republic of Korea.
}

\begin{abstract}
Studies on degenerate versions of Stirling, Bernoulli and Eulerian numbers started by [L. Carlitz, Utilitas Math., 15 (1979), 51-88]. In recent years, many mathematicians have studied degenerate version of various special polynomials and numbers. In this paper, we introduce the q-analogue degenerate Carlitz's type Daehee and higher-order degenerate Carlitz's type Daehee polynomials and numbers. Also, we study some explicit identities and properties for the q-analogue degenerate Carlitz's type Daehee polynomials and numbers and higher-order q-analogue degenerate Carlitz's type Daehee polynomials and numbers arising from $p$-adic invariant $q$-integral on $\mathbb{Z}_{\mathrm{p}}$.
\end{abstract}

Keywords: p-Adic q-integral of $f$ on $\mathbb{Z}_{p}$, degenerate Carlitz's type Daehee polynomials and numbers, degenerate $q$-Bernoulli polynomials.

2010 MSC: 11B68, 11S40, 11S80.

(C)2019 All rights reserved.

\section{Introduction}

Let $p$ be a fixed prime number. Throughout this paper, $\mathbb{Z}_{p}, \mathbb{Q}_{p}$, and $\mathbb{C}_{p}$ will denote the ring of $p$-adic integers, the field of $p$-adic rational numbers, and the completion of algebraic closure of $\mathbb{Q}_{p}$, respectively. The $p$-adic norm $|\cdot|_{p}$ is normalized as $|p|_{p}=\frac{1}{p}$. Let $q$ be in $\mathbb{C}_{p}$ with $|q-1|_{p}<p^{-\frac{1}{p-1}}$ and $x$ in $\mathbb{Z}_{p}$. Then the $\mathrm{q}$-analogue of $\mathrm{x}$ is defined to be $[x]_{\mathrm{q}}=\frac{1-\mathrm{q}^{\mathrm{x}}}{1-\mathrm{q}}$. Note that $\lim _{\mathrm{q} \rightarrow 1}[\mathrm{x}]_{\mathrm{q}}=\mathrm{x}$.

For $f \in U D\left(\mathbb{Z}_{p}\right)$, the space of uniformly differentiable functions on $\mathbb{Z}_{p}$, the $p$-adic q-integral of $f$ on $\mathbb{Z}_{p}$ is defined by Kim to be (see [1, 4, 6, 7, 15-17])

$$
I_{q}(f)=\int_{\mathbb{Z}_{p}} f(x) d \mu_{q}(x)=\lim _{N \rightarrow \infty} \frac{1}{\left[p^{N}\right]_{q}} \sum_{n=0}^{p^{N}-1} f(x) q^{x} .
$$

\footnotetext{
*Corresponding author

Email addresses: mathkjk26@gnu.ac.kr (Jongkyum Kwon), kimholzi@gmail.com (Yunjae Kim), gysohn@dnue.ac.kr (Gyoyong Sohn), a0417001@knu.ac.kr (Jin-Woo Park)
}

doi: $10.22436 /$ jmcs.019.02.08

Received: 2019-04-13 Revised: 2019-04-28 Accepted: 2019-05-02 
From (1.1), we can drive the following integral identity.

$$
q I_{q}\left(f_{1}\right)-I_{q}(f)=\frac{q-1}{\log q} f^{\prime}(0)+(q-1) f(0),
$$

where $f_{1}(x)=f(x+1)$. Also, the Carlitz's q-Bernoulli polynomials $\beta_{n, q}(x)$ are defined to be

$$
\beta_{n, q}(x)=\sum_{l=0}^{n}\left(\begin{array}{l}
n \\
l
\end{array}\right) q^{l x}[x]_{q}^{n-l} \beta_{l, q}
$$

where

$$
\beta_{n, q}=\int_{\mathbb{Z}_{p}}[y]_{q}^{n} d \mu_{q}(y),(n \geqslant 0), \quad(\text { see }[2,3,8,12])
$$

Indeed,

$$
[x+y]_{q}^{n}=\left(\frac{1-q^{x}+q^{x}\left(1-q^{y}\right)}{1-q}\right)^{n}=\left([x]_{q}+q^{x}[y]_{q}\right)^{n}=\sum_{l=0}^{n}\left(\begin{array}{c}
n \\
l
\end{array}\right) q^{l x}[y]_{q}^{l}[x]_{q}^{n-l}
$$

By (1.2) and (1.3), the Carlitz's q-Bernoulli polynomials can be represented by p-adic q-integral on $\mathbb{Z}_{\mathrm{p}}$ as follows:

$$
\beta_{n, q}(x)=\sum_{l=0}^{n}\left(\begin{array}{c}
n \\
l
\end{array}\right) q^{l x}[x]_{q}^{n-l} \beta_{l, q}=\sum_{l=0}^{n}\left(\begin{array}{l}
n \\
l
\end{array}\right) q^{l x}[x]_{q}^{n-l} \int_{\mathbb{Z}_{p}}[y]_{q}^{l} d u_{q}(y)=\int_{\mathbb{Z}_{p}}[x+y]_{q}^{n} d u_{q}(y) .
$$

The Carlitz's type degenerate q-Bernoulli polynomials can be represented by p-adic q-integral on $\mathbb{Z}_{p}$ as follows:

$$
\int_{\mathbb{Z}_{\mathrm{p}}}(1+\lambda t)^{\frac{[x+y]_{\mathrm{q}}}{\lambda}} \mathrm{d} \mu_{\mathrm{q}}(\mathrm{y})=\sum_{n=0}^{\infty} B_{n, q}(x \mid \lambda) \frac{\mathrm{t}^{n}}{n !}, \quad \text { (see [9]). }
$$

The Daehee polynomials are defined by the generating function to be

$$
\frac{\log (1+t)}{t}(1+t)^{x}=\sum_{n=0}^{\infty} D_{n}(x) \frac{t^{n}}{n !}, \quad(\text { see }[4,9-11,13,14,19]) .
$$

In the special case $x=0, D_{n}=D_{n}(0)$ are called the Daehee numbers.

The degenerate Daehee polynomials are given by the generating function to be

$$
\frac{\lambda \log \left(1+\frac{1}{\lambda} \log (1+\lambda t)\right)}{\log (1+\lambda t)}\left(1+\frac{1}{\lambda} \log (1+\lambda t)\right)^{x}=\sum_{n=0}^{\infty} D_{n, \lambda}(x) \frac{t^{n}}{n !}, \quad \text { (see [14]). }
$$

For $x=0, D_{n, \lambda}=D_{n, \lambda}(0)$ are called the degenerate Daehee numbers. We observe here that $D_{n, \lambda} \longrightarrow D_{n}$ as $\lambda \longrightarrow 0$.

The Stirling numbers of the first kind are given by

$$
(x)_{n}=\sum_{l=0}^{n} S_{1}(n, l) x^{l}(x \geqslant 0),(\text { see }[3,20]),
$$

where $(x)_{n}=x(x-1) \cdots(x-n+1)$ is the $n$ falling factorial, and the Stirling numbers of the first kind are defined by the generating function to be

$$
(\log (x+1))^{n}=n ! \sum_{l=n}^{\infty} S_{1}(l, n) \frac{x^{l}}{l !},(n \geqslant 0),(\text { see }[5,18]) .
$$

Recently, several researchers have studied degenerate type special polynomials related to Bernoulli, Euler, Daehee, and Changhee polynomials. In this paper, we introduce the q-analogue degenerate Carlitz's type Daehee polynomials and numbers and q-analogue higher-order degenerate Carlitz's type Daehee polynomials. Also, we study some explicit identities and properties for those polynomials arising from $\mathrm{p}$-adic invariant $\mathrm{q}$-integral on $\mathbb{Z}_{\mathrm{p}}$. 


\section{The q-analogue degenerate Carlitz's type Daehee polynomials and numbers}

In this section, we assume that $\lambda, t \in \mathbb{C}_{p}$ with $|\lambda t|_{p}<p^{-\frac{1}{p-1}}$ and $q \in \mathbb{C}_{p}$ with $|1-q|_{p}<p^{-\frac{1}{p-1}}$.

In the viewpoint of (1.2), we define the q-analogue degenerate Carlitz's type Daehee polynomials which are given by the generating function to be

$$
\int_{\mathbb{Z}_{p}}(1+\lambda \log (1+t))^{\frac{[x+y]_{q}}{\lambda}} \mathrm{d} \mu_{q}(y)=\sum_{n=0}^{\infty} D_{n, q, \lambda}(x) \frac{t^{n}}{n !}, \quad(n \geqslant 0) .
$$

When $x=0, D_{n, q, \lambda}=D_{n, q, \lambda}(0)$ are called the $q$-analogue degenerate Carlitz's type Daehee numbers.

From (2.1), we note that

$$
\begin{aligned}
\sum_{n=0}^{\infty} D_{n, q, \lambda}(x) \frac{t^{n}}{n !} & =\int_{\mathbb{Z}_{p}}(1+\lambda \log (1+t))^{\frac{[x+y]_{q}}{\lambda}} d \mu_{q}(y) \\
& =\int_{\mathbb{Z}_{p}} \sum_{k=0}^{\infty}\left(\begin{array}{c}
\frac{[x+y]_{q}}{\lambda} \\
k
\end{array}\right) \lambda^{k}(\log (1+t))^{k} d \mu_{q}(y) \\
& =\sum_{k=0}^{\infty} \int_{\mathbb{Z}_{p}}\left(\frac{[x+y]_{q}}{\lambda}\right)_{k} \lambda^{k} \frac{1}{k !}(\log (1+t))^{k} d \mu_{q}(y) \\
& =\sum_{k=0}^{\infty} \int_{\mathbb{Z}_{p}}\left([x+y]_{q}\right)_{k, \lambda} \sum_{n=k}^{\infty} S_{1}(n, k) d \mu_{q}(y) \frac{t^{n}}{n !} \\
& =\sum_{n=0}^{\infty} \int_{\mathbb{Z}_{p}} \sum_{k=0}^{n}\left([x+y]_{q}\right)_{k, \lambda} S_{1}(n, k) d \mu_{q}(y) \frac{t^{n}}{n !}
\end{aligned}
$$

where $\left([x+y]_{q}\right)_{k, \lambda}=[x+y]_{q}\left([x+y]_{q}-\lambda\right) \cdots\left([x+y]_{q}-(k-1) \lambda\right)$.

Comparing the coefficients of $\frac{\mathrm{t}^{\mathrm{n}}}{n !}$ on the both sides of (2.2), we have the following theorem.

Theorem 2.1. For $\mathrm{n} \geqslant 0$, we have

$$
D_{n, q, \lambda}(x)=\sum_{k=0}^{n} \int_{\mathbb{Z}_{p}}\left([x+y]_{q}\right)_{k, \lambda} s_{1}(n, k) d \mu_{q}(y) .
$$

By replacing $t$ by $e^{t}-1$ in (2.1), we obtain

$$
\sum_{m=0}^{\infty} D_{m, q, \lambda}(x) \frac{1}{m !}\left(e^{t}-1\right)^{m}=\int_{\mathbb{Z}_{p}}(1+\lambda t)^{\frac{[x+y]_{q}}{\lambda}} d \mu_{q}(y)=\sum_{m=0}^{\infty} B_{m, q}(x \mid \lambda) \frac{t^{n}}{n !} .
$$

On the other hand, we observe the left hand side of the previous equation.

$$
\sum_{k=0}^{\infty} D_{k, q, \lambda}(x) \frac{1}{k !}\left(e^{t}-1\right)^{k}=\sum_{k=0}^{\infty} D_{k, q, \lambda}(x) \sum_{m=k}^{\infty} S_{2}(m, k) \frac{t^{m}}{m !}=\sum_{m=0}^{\infty}\left(\sum_{k=0}^{m} D_{k, q, \lambda}(x) S_{2}(m, k)\right) \frac{t^{m}}{m !} .
$$

Comparing the coefficients of $\frac{\mathrm{t}^{\mathrm{m}}}{\mathrm{m} !}$ on the both sides of (2.3) and (2.4), we arrive at the following theorem.

Theorem 2.2. For $\mathrm{n} \geqslant 0$, we have

$$
B_{m, q}(x \mid \lambda)=\sum_{k=0}^{m} D_{k, q, \lambda}(x) S_{2}(m, k)
$$


Now, we consider the inversion formula of Theorem 2.2. By replacing $t$ by $\log (1+t)$ in $(1.4)$, we obtain

$$
\begin{aligned}
\int_{\mathbb{Z}_{p}}(1+\lambda \log (1+t))^{\frac{[x+y]_{q}}{\lambda}} \mathrm{d} \mu_{q}(y) & =\sum_{m=0}^{\infty} B_{m, q}(x \mid \lambda) \frac{1}{m !}(\log (1+t))^{m} \\
& =\sum_{m=0}^{\infty} B_{m, q}(x \mid \lambda) \sum_{n=m}^{\infty} S_{1}(n, m) \frac{t^{n}}{n !} \\
& =\sum_{n=0}^{\infty}\left(\sum_{m=0}^{n} B_{m, q}(x \mid \lambda) S_{1}(n, m)\right) \frac{t^{n}}{n !}
\end{aligned}
$$

On the other hand, we observe the left hand side of the previous equation.

$$
\int_{\mathbb{Z}_{p}}(1+\lambda \log (1+t))^{\frac{[x+y]_{q}}{\lambda}} d \mu_{q}(y)=\sum_{m=0}^{\infty} D_{m, q, \lambda}(x) \frac{t^{m}}{m !} .
$$

Theorem 2.3. For $m \geqslant 0$, we have

$$
D_{m, q, \lambda}(x)=\sum_{m=0}^{n} B_{m, q}(x \mid \lambda) S_{1}(n, m)
$$

Now, we observe that

$$
\begin{aligned}
(1+\lambda \log (1+\mathrm{t}))^{\frac{[x+y]_{\mathrm{q}}}{\lambda}} & =e^{\frac{[x+y]_{\mathrm{q}}}{\lambda} \log (1+\lambda \log (1+\mathrm{t}))} \\
& =\sum_{n=0}^{\infty}\left(\frac{[x+y]_{\mathrm{q}}}{\lambda}\right)^{n} \frac{1}{n !}(\log (1+\lambda \log (1+\mathrm{t})))^{n} \\
& =\sum_{n=0}^{\infty}\left(\frac{[x+y]_{\mathrm{q}}}{\lambda}\right)^{n} \sum_{m=n}^{\infty} S_{1}(m, n) \lambda^{m} \frac{(\log (1+t))^{m}}{m !} \\
& =\sum_{k=0}^{\infty}\left(\sum_{m=0}^{k} \sum_{n=0}^{m}[x+y]_{q}^{n} \lambda^{m-n} S_{1}(m, n) S_{1}(k, m)\right) \frac{t^{k}}{k !} .
\end{aligned}
$$

From (2.5), we obtain

$$
\begin{aligned}
\sum_{k=0}^{\infty} D_{k, q, \lambda}(x) \frac{t^{k}}{k !} & =\int_{\mathbb{Z}_{p}}(1+\lambda \log (1+t))^{\frac{[x+y]_{q}}{\lambda}} d \mu_{q}(y) \\
& =\int_{\mathbb{Z}_{p}} \sum_{k=0}^{\infty}\left(\sum_{m=0}^{k} \sum_{n=0}^{m}[x+y]_{q}^{n} \lambda^{m-n} S_{1}(m, n) S_{1}(k, m)\right) d \mu_{q}(y) \frac{t^{k}}{k !} \\
& =\sum_{k=0}^{\infty}\left(\sum_{m=0}^{k} \sum_{n=0}^{m} \lambda^{m-n} S_{1}(m, n) S_{1}(k, m) B_{n, q}(x)\right) \frac{t^{k}}{k !} .
\end{aligned}
$$

Comparing the coefficients of $\frac{\mathrm{t}^{\mathrm{k}}}{\mathrm{k} !}$ on the both sides of (2.6), we arrive at the following theorem.

Theorem 2.4. For $k \geqslant 0$, we have

$$
D_{k, q, \lambda}(x)=\sum_{m=0}^{k} \sum_{n=0}^{m} \lambda^{m-n} S_{1}(m, n) S_{1}(k, m) B_{n, q}(x) .
$$


Note that

$$
[x+y]_{q}=\frac{1-q^{x+y}}{1-q}=\frac{1-q^{x}}{1-q}+\frac{q^{x}\left(1-q^{y}\right)}{1-q}=[x]_{q}+q^{x}[y]_{q}
$$

We observe that

$$
\begin{aligned}
(1+\lambda & \log (1+t))^{\frac{[x+y]_{q}}{\lambda}} \\
& =(1+\lambda \log (1+t))^{\frac{[x]_{q}}{\lambda}}(1+\lambda \log (1+t))^{\frac{q^{x}[y]_{q}}{\lambda}} \\
& =\left(\sum_{m=0}^{\infty}\left(\frac{[x]_{q}}{\lambda}\right)_{m} \lambda^{m} \frac{(\log (1+t))^{m}}{m !}\right)\left(\sum_{l=0}^{\infty} \frac{q^{l x}[y]_{q}^{l}}{\lambda^{l}} \frac{\left(\log (1+\lambda \log (1+t))^{l}\right.}{l !}\right) \\
& =\left(\sum_{k=0}^{\infty}\left(\sum_{m=0}^{k}\left([x]_{q}\right)_{m, \lambda} S_{1}(n, m)\right) \frac{t^{k}}{k !}\right)\left(\sum_{j=0}^{\infty}\left(\sum_{p=0}^{j} \sum_{l=0}^{p} \lambda^{p-l} q^{l x}[y]_{q}^{l} S_{1}(p, l) S_{1}(j, p)\right) \frac{t^{j}}{j !}\right) \\
& =\sum_{n=0}^{\infty}\left(\sum_{j=0}^{n} \sum_{m=0}^{n-j} \sum_{p=0}^{j} \sum_{l=0}^{p}\left(\begin{array}{l}
n \\
j
\end{array}\right)\left([x]_{q}\right)_{m, \lambda} \lambda^{p-l} q^{l x}[y]_{q}^{l} S_{1}(m, n) S_{1}(p, l) S_{1}(j, p)\right) \frac{t^{n}}{n !} .
\end{aligned}
$$

Comparing the coefficients of $\frac{\mathrm{t}^{\mathrm{n}}}{\mathrm{n} !}$ on the both sides of (2.6), we arrive at the following theorem.

Theorem 2.5. For $\mathrm{n} \geqslant 0$, we have

$$
D_{n, q, \lambda}(x)=\sum_{j=0}^{n} \sum_{m=0}^{n-j} \sum_{p=0}^{j} \sum_{l=0}^{p}\left(\begin{array}{l}
n \\
j
\end{array}\right)\left([x]_{q}\right)_{m, \lambda} \lambda^{p-l} q^{l x}[y]_{q}^{l} S_{1}(m, n) S_{1}(p, l) S_{1}(j, p) .
$$

Using (2.4), we get

$$
\begin{aligned}
\sum_{n=0}^{\infty} D_{n, q, \lambda}(x) \frac{t^{n}}{n !}= & \int_{\mathbb{Z}_{p}}(1+\lambda \log (1+t))^{\frac{[x+y]_{q}}{\lambda}} d \mu_{q}(y) \\
= & \lim _{N \rightarrow \infty} \frac{1}{\left[p^{N}\right]_{q}} \sum_{y=0}^{p^{N}-1}(1+\lambda \log (1+t))^{\frac{[x+y]_{q}}{\lambda}} q^{y} \\
= & \lim _{N \rightarrow \infty} \frac{1}{\left[d p^{N}\right]_{q}} \sum_{a=0}^{d-1} \sum_{y=0}^{p^{N}-1}(1+\lambda \log (1+t))^{\frac{[x+a+d y]_{q}}{\lambda}} q^{a+d y} \\
= & \lim _{N \rightarrow \infty} \frac{1}{[d]_{q}} \frac{1}{\left[p^{N}\right]_{q^{d}}} \sum_{a=0}^{d-1} \sum_{y=0}^{N-1}(1+\lambda \log (1+t))^{\frac{[d]_{q}\left[\frac{a+x}{d}+y\right]_{q^{d}}}{\lambda}} q^{a}\left(q^{d}\right)^{y} \\
= & \frac{1}{[d]_{q}} \sum_{a=0}^{d-1} q^{a} \lim _{N \rightarrow \infty} \frac{1}{\left[p^{N}\right]_{q^{d}}} \sum_{y=0}^{p^{N}-1} \sum_{l=0}^{\infty}\left(\frac{[d]_{q}\left[\frac{a+x}{d}+y\right]_{q^{d}}}{\lambda}\right) \lambda^{l}(\log (1+t))^{l}\left(q^{d}\right)^{y} \\
= & \frac{1}{[d]_{q}} \sum_{a=0}^{d-1}(q)^{a} \lim _{N \rightarrow \infty} \frac{1}{\left[p^{N}\right]_{q^{d}}} \\
& \times \sum_{y=0}^{p^{N}-1} \sum_{n=0}^{\infty}\left(\sum_{l=0}^{n} \sum_{k=0}^{l} S_{1}(l, k) S_{1}(n, l)[d]_{q}^{k}\left[\frac{a+x}{d}+y\right]_{q^{d}}^{k} \lambda^{n-l}\right) \frac{t^{n}}{n !}\left(q^{d}\right)^{y} \\
= & \sum_{n=0}^{\infty}\left(\sum_{l=0}^{n} \sum_{k=0}^{l} \frac{1}{[d]_{q}} \sum_{a=0}^{d-1}(q)^{a} S_{1}(l, k) S_{1}(n, l)[d]_{q}^{k} \lambda^{n-l} B_{k, q^{d}}\left(\frac{a+x}{d}\right)\right) \frac{t^{n}}{n !}
\end{aligned}
$$

Comparing the coefficients of $\frac{\mathrm{t}^{\mathrm{n}}}{\mathrm{n} !}$ on the both sides of (2.7), we arrive at the following theorem. 
Theorem 2.6. For $\mathrm{n} \geqslant 0$, we have

$$
D_{n, q, \lambda}(x)=\sum_{l=0}^{n} \sum_{k=0}^{l} \frac{1}{[d]_{q}} \sum_{a=0}^{d-1}(q)^{a} S_{1}(l, k) S_{1}(n, l)[d]_{q}^{k} \lambda^{n-l} B_{k, q d}\left(\frac{a+x}{d}\right) .
$$

For $r \in \mathbb{N}$, the higher-order q-analogue degenerate Carlitz's type Daehee polynomials are given by the multivariate bosonic $p$-adic q-integral as follows:

$$
\int_{\mathbb{Z}_{p}} \cdots \int_{\mathbb{Z}_{p}}(1+\lambda \log (1+t))^{\frac{\left[x_{1}+\cdots+x_{r}+x\right]_{q}}{\lambda}} d \mu_{q}\left(x_{1}\right) \cdots d \mu_{q}\left(x_{r}\right)=\sum_{n=0}^{\infty} D_{n, q, \lambda}^{(r)}(x) \frac{t^{n}}{n !}, \quad(n \geqslant 0) .
$$

Now, we observe that

$$
\begin{aligned}
& \sum_{n=0}^{\infty} D_{n, q, \lambda}^{(r)}(x) \frac{t^{n}}{n !} \\
& \quad=\int_{\mathbb{Z}_{p}} \cdots \int_{\mathbb{Z}_{p}}(1+\lambda \log (1+t))^{\frac{\left[x_{1}+\cdots+x_{r}+x\right]_{q}}{\lambda}} d \mu_{q}\left(x_{1}\right) \cdots d \mu_{q}\left(x_{r}\right) \\
& \quad=\int_{\mathbb{Z}_{p}} \cdots \int_{\mathbb{Z}_{p}} \sum_{l=0}^{\infty}\left(\frac{\left[x_{1}+\cdots+x_{r}+x\right]_{q}}{\lambda}\right)(\lambda \log (1+t))^{l} d \mu_{q}\left(x_{1}\right) \cdots d \mu_{q}\left(x_{r}\right) \\
& =\int_{\mathbb{Z}_{p}} \cdots \int_{\mathbb{Z}_{p}} \sum_{l=0}^{\infty}\left(\left[x_{1}+\cdots+x_{r}+x\right]_{q}\right)_{l, \lambda} \frac{1}{l !}(\log (1+t))^{l} d \mu_{q}\left(x_{1}\right) \cdots d \mu_{q}\left(x_{r}\right) \\
& =\int_{\mathbb{Z}_{p}} \cdots \int_{\mathbb{Z}_{p}} \sum_{n=0}^{\infty}\left(\sum_{l=0}^{n}\left(\left[x_{1}+\cdots+x_{r}+x\right]_{q}\right)_{l, \lambda} S_{1}(n, l)\right) d \mu_{q}\left(x_{1}\right) \cdots d \mu_{q}\left(x_{r}\right) \\
& =\int_{\mathbb{Z}_{p}} \cdots \int_{\mathbb{Z}_{p}} \sum_{n=0}^{\infty}\left(\sum_{l=0}^{n} \sum_{k=0}^{l} S_{1}(l, k)\left[x_{1}+\cdots+x_{r}+x\right]_{q}^{k} \lambda^{l-k} S_{1}(n, l)\right) d \mu_{q}\left(x_{1}\right) \cdots d \mu_{q}\left(x_{r}\right) \frac{t^{n}}{n !} \\
& =\sum_{n=0}^{\infty}\left(\sum_{l=0}^{n} \sum_{k=0}^{l} S_{1}(l, k) S_{1}(n, l) \lambda^{l-k} B_{k, q}^{(r)}(x)\right) \frac{t^{n}}{n !} .
\end{aligned}
$$

Comparing the coefficients of $\frac{\mathrm{t}^{\mathrm{n}}}{\mathrm{n} !}$ on the both sides of (2.9), we arrive at the following theorem.

Theorem 2.7. For $\mathrm{n} \geqslant 0$, we have

$$
D_{n, q, \lambda}^{(r)}(x)=\sum_{l=0}^{n} \sum_{k=0}^{l} S_{1}(l, k) S_{1}(n, l) \lambda^{l-k} B_{k, q}^{(r)}(x) .
$$

Substitute $t$ by $e^{t}-1$ in (2.8), we get

$$
\sum_{k=0}^{\infty} D_{k, q, \lambda}^{(r)}(x) \frac{1}{k !}\left(e^{t}-1\right)^{k}=\int_{\mathbb{Z}_{p}} \cdots \int_{\mathbb{Z}_{p}}(1+\lambda t)^{\frac{\left[x_{1}+\cdots+x_{r}+x\right]_{q}}{\lambda}} d \mu_{q}\left(x_{1}\right) \cdots d \mu_{q}\left(x_{r}\right)=\sum_{n=0}^{\infty} B_{k, q}^{(r)}(x \mid \lambda) \frac{t^{n}}{n !} .
$$

On the other hand, we observe the left hand side of the previous equation.

$$
\sum_{k=0}^{\infty} D_{k, q, \lambda}^{(r)}(x) \frac{1}{k !}\left(e^{t}-1\right)^{k}=\sum_{k=0}^{\infty} D_{k, q, \lambda}^{(r)}(x) \sum_{n=k}^{\infty} S_{2}(n, k) \frac{t^{n}}{n !}=\sum_{n=0}^{\infty}\left(\sum_{k=0}^{n} D_{k, q, \lambda}^{(r)}(x) S_{2}(n, k)\right) \frac{t^{n}}{n !} .
$$

Comparing the coefficients of $\frac{t^{n}}{n !}$ on the both sides of (2.10) and (2.11), we arrive at the following theorem.

Theorem 2.8. For $\mathrm{n} \geqslant 0$, we have

$$
B_{k, q}^{(r)}(x \mid \lambda)=\sum_{k=0}^{n} D_{k, q, \lambda}^{(r)}(x) S_{2}(n, k) .
$$




\section{Acknowledgment}

The authors would like to thank the referees for many valuable and detailed comments.

\section{References}

[1] A. Bayad, T. Kim, Identities involving values of Bernstein, q-Bernoulli, and q-Euler polynomials, Russ. J. Math. Phys., 18 (2011), 133-143. 1

[2] L. Carlitz, Expansions of q-Bernoulli numbers, Duke Math. J., 25 (1958), 355-364. 1

[3] L. Carlitz, Degenerate Stirling, Bernoulli and Eulerian numbers, Utilitas Math., 15 (1979), 51-88. 1, 1

[4] Y.-H. Cho, T. Kim, T. Mansour, S.-H. Rim, Higher-order q-Daehee polynomials, J. Comput. Anal. Appl., 19 (2015), 167-173. 1,1

[5] L. Comtet, Advanced Combinatorics, D. Reidel Publishing Co., Dordrecht, (1974). 1

[6] T. Kim, q-Volkenborn integration, Russ. J. Math. Phys., 9 (2002), 288-299. 1

[7] T. Kim, Some formulae for the q-Bernoulli and Euler polynomials of higher order, J. Math. Anal. Appl., 273 (2002), 236-242. 1

[8] T. Kim, Barnes' type multiple degenerate Bernoulli and Euler polynomials, Appl. Math. Comput., 258 (2015), 556-564. 1

[9] T. Kim, On Degenerate q-Bernoulli polynomials, Bull. Korean Math. Soc., 53 (2016), 1149-1156. 1.4, 1

[10] D. S. Kim, T. Kim, Daehee numbers and polynomials, Appl. Math. Sci. (Ruse), 7 (2013), 5969-5976.

[11] D. S. Kim, T. Kim, Identities arising from higher-order Daehee polynomials bases, Open Math., 13 (2015), 196-208. 1

[12] T. Kim, D. S. Kim, H.-I. Kwon, Some identities of Carlitz degenerate Bernoulli numbers and polynomials, Iran. J. Sci. Technol. Trans. A Sci., 41 (2017), 749-753. 1

[13] D. S. Kim, T. Kim, S.-H. Lee, J.-J. Seo, Higher-order Daehee numbers and polynomials, Int. J. Math. Anal. (Ruse), 8 (2014), 273-283. 1

[14] D. Lim, Degenerate, partially degenerate and totally degenerate Daehee numbers and polynomials, Adv. Difference Equ., 2015 (2015), 14 pages. 1

[15] D. Lim, Modified q-Daehee numbers and polynomials, J. Comput. Anal. Appl., 21 (2016), 324-330. 1

[16] H. Ozden, I. N. Cangul, Y. Simsek, Remark on q-Bernoulli numbers associated with Daehee numbers, Adv. Stud. Contemp. Math. (Kyungshang), 18 (2009), 41-48.

[17] J.-W. Park, On the twisted Daehee polynomialss with q-parameter, Adv. Difference Equ., 2014 (2014), 10 pages. 1

[18] S. Roman, The umbral calculus, Dover, New York, (2005). 1

[19] J.-J. Seo, T. Kim, Some identities of symmetry for Daehee polynomials arising from $\mathbf{p}$-adic invariant integral on $\mathbb{Z}_{\mathbf{p}}$, Proc. Jangjeon Math. Soc., 11 (2008), 191-198. 1

[20] N. L. Wang, Some identities involving generalized Bernoulli numbers (Chinese), J. Inn. Mong. Norm. Univ. Nat. Sci., 43 (2014), 403-407. 1 